\title{
Die Rolle der Gutachterkommissionen und Schlichtungsstellen in der Qualitätssicherung
}

Die Gutachterkommission für ärztliche Behandlungsfehler bei der Ärztekammer Nordrhein in Düsseldorf wurde 1975 auf Initiative der Ärztekammer eingerichtet, um auf Antrag zu prüfen, ob ein vorwerfbarer ärztlicher Behandlungsfehler vorliegt, der einen Gesundheitsschaden verursacht hat. Eine Stellungnahme (Bescheid), z.T. auf ein Gutachten gestützt, an den Patienten und den Arzt stellt die Grundlage für einen außergerichtlichen Vergleich mit den Versicherungsgesellschaften dar. Seit der Wiedervereinigung bestehen in allen Ländern der Bundesrepublik entsprechende Gutachterkommissionen/Schlichtungsstellen, die in der von der Bundesärztekammer eingerichteten Ständigen Konferenz ihre Ergebnisse austauschen. Danach kommen nach einer Zusammenstellung der Jahre bis Ende 2003 etwa 60\% der Anträge wegen eines vermeintlichen Behandlungsfehlers aus den operativen Fächern, davon 13\% aus der Gynäkologie und Geburtshilfe; aus den Gebieten der Inneren Medizin 9\% (Tab.1). Die Behandlungsfehlerquote beträgt mit geringen Unterschieden in den letzten Jahren etwa 33\% (Tab.1).

In den vergangenen Jahren führten bis zu 90\% der gutachtlichen Bescheide zu einer außergerichtlichen Streitschlichtung; gut 10\% der betroffenen Ärzte und Patienten waren mit dem gutachtlichen Bescheid nicht zufrieden und gingen vor Gericht, wobei etwa $1 \%$ von der Beurteilung der Gutachterkommission abweichende Gerichtsentscheidungen ergangen sind.

Danach dienen die Gutachterkommissionen/Schlichtungsstellen in Deutschland in erster Linie einem verbesserten Arzt-/Patientenverhältnis im Sinne einer befriedenden Wirkung. Die Dauer der für die Beteiligten kostenfreien Verfahren ist im Vergleich zu Gerichtsverfahren mit durchschnittlich 12 - 15 Monaten deutlich kürzer. Aufgrund der hohen Akzeptanz der Bescheide bei den Versicherungsgesellschaften führen die außergerichtlichen Schlichtungsverfahren im Falle der Bejahung von Behandlungsfehlern mit kausalem Gesundheitsschaden in der Regel zum Erfolg im Sinne einer Regulierung der Schadensersatz- und Schmerzensgeldansprüche. Sie dienen gleichfalls der Abwehr unbegründeter Ansprüche für den in Anspruch genommenen Arzt und bewirken eine Klärung der beide Seiten belastenden Vorwürfe.

Aus den Ergebnissen der Gutachterkommissionen/Schlichtungsstellen ist aber aufgrund der fehlenden Kenntnisse über die Gesamtzahl der ärztlichen Behandlungen in einer vorgegebenen Zeit keine sichere Aussage über die Schadens- und Fehlerrate möglich (die Schadensrate gibt den Prozentsatz der ärztlichen verursachten Schäden im Vergleich zur Gesamtheit der Behandlungsfälle pro Fachgebiet wider, während die Fehlerrate den Prozentsatz der durch ärztliche Sorgfaltsmängel verursachten Schäden angibt).

Die Ergebnisse der Gutachterkommissionen/Schlichtungsstellen lassen sich auch zur Lösung von Qualitätsproblemen durch Auswertung der Gutachten und Bescheide heranziehen:

1. Bei fehlerhafter Indikation und Durchführung einer Operation (Risiko- und Eingriffsaufklärung).

2. Im Falle eines fehlerhaften Befundes oder einer Diagnose (Befund- und Diagnoseaufklärung).

3. Durch Fehler einer therapeutisch gebotenen Aufklärung zur Gefahrenabwehr, um den Patienten durch eine entsprechende Information zur Dringlichkeit einer gebotenen Behandlung oder Untersuchung zu veranlassen (Risiko- und Eingriffsaufklärung). Dabei bedeutet das Fehlen einer Sicherungsaufklärung ein ärztlicher Behandlungsfehler. 
Tab. 1

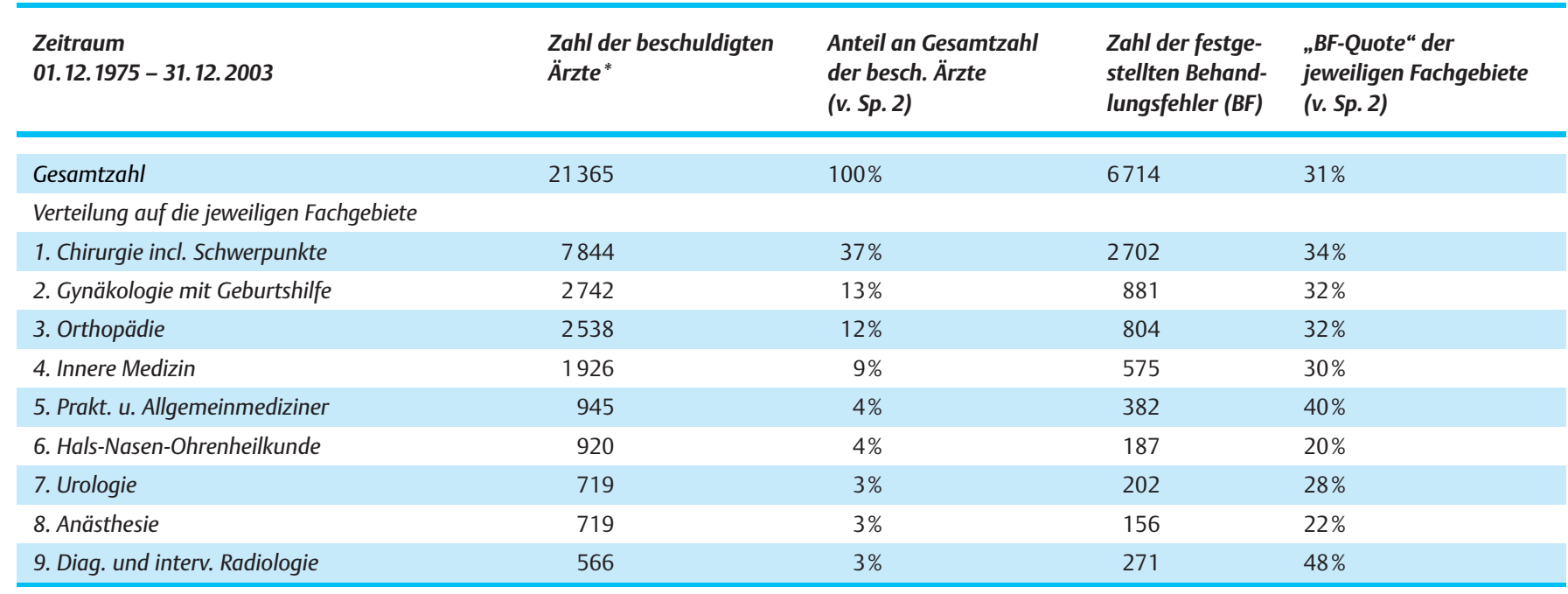

* Mehrfachnennung pro Verfahren: nur 1 Arzt pro in Anspruch genommener Einrichtung (Klinikabteilung/Praxis)

Entwicklung der Behandlungsfehlervorwürfe

in der Gynäkologie und Geburtshilfe im letzten Jahrzehnt

in der Gutachterkommission

bei der Ärztekammer Nordrhein

Anträge aus den chirurgischen Fachgebieten Allgemeine und Unfallchirurgie, Orthopädie und Gynäkologie mit Geburtshilfe machen über die Jahre konstant zwei Drittel aller Verfahren bei der Gutachterkommission aus, wobei die Gynäkologie mit einem Anteil von $13 \%$ vor der Orthopädie (12\%) das am zweithäufigsten betroffene Fachgebiet darstellt. Insgesamt entfallen etwa $2 / 3$ der Behandlungsfehlervorwürfe auf im Krankenhaus tätige Ärzte. Von 741 gutachtlichen Bescheiden im Fachgebiet Gynäkologie und Geburtshilfe der letzten 5 Jahre entfielen wie bisher $1 / 3$ auf Vorwürfe in der Geburtshilfe. Den im Krankenhaus tätigen Gynäkologen wurden in $85 \%$ Fehler bei operativen Maßnahmen sowie bei der Nachbehandlung vorgeworfen. Mit jeweils einem Viertel standen hier die Mammachirurgie und die laparoskopischen Eingriffe, gefolgt von den abdominalen (12\%) und den vaginalen (11\%) Hysterektomien im Vordergrund der Vorwürfe. Fast $60 \%$ der anerkannten Behandlungsfehler in der Gynäkologie ereignen sich im Rahmen der operativen Therapie mit in etwa gleich hohen Behandlungsfehlerquoten (36-38\%). Allein bei der radikalen Hysterektomie (5\%) und den kosmetischen Eingriffen (3\%), die nicht Teil der Mammachirurgie waren, war die Anerkennungsquote mit $47 \%$ bzw. $50 \%$ höher. Bezogen auf alle festgestellten Fehler standen im Vordergrund intraoperative $(43 \%)$ und Fehler in der Nachbetreuung (31\%), gefolgt von der fehlenden Indikation (14\%) und der Verfahrenswahl (9\%); präoperative Mängel wurden selten festgestellt (3\%). Häufige Fehler waren bei den Hysterektomien und den laparoskopischen Eingriffen die Verkennung von Ureterläsionen sowie von Läsionen des MagenDarm-Traktes im Rahmen der laparoskopischen Adhäsiolyse, in der Mammachirurgie die unzureichende PE.

Niedergelassenen Gynäkologen wurden in der Hälfte der Bescheide diagnostische Versäumnisse vorgeworfen, wie die Ver- kennung bösartiger Tumoren, besonders des Mammakarzinoms ( $2 / 3$ der Vorwürfe). Die Anerkennungsquoten lagen deutlich über dem Durchschnitt, insbesondere beim Mammakarzinom (59\%) und Cervixkarzinom (46\%). Der Vorwurf der Verkennung einer Extrauteringravidität war in der Hälfte der diesbezüglich geführten Verfahren berechtigt. Der bezogen auf die Gesamtheit der Begutachtungsverfahren selten erhobene Vorwurf $(4 \%$ ) des Eintritts einer Paravasation bei der Verabreichung von Chemotherapeutika betraf ganz überwiegend die onkologisch tätigen Gynäkologen (insbesondere durch Epirubicin). Dieser für die Patienten oftmals schwerwiegenden Komplikation kommt auch im Hinblick auf die erhöhte Quote dabei festgestellter Behandlungsfehler ( $45 \%$ ) eine nicht zu vernachlässigende Bedeutung für die Qualitätssicherung zu.

Der Anteil der in den letzten 5 Jahren gegen Krankenhausbehandlungen erhobenen Vorwürfe in der Geburtshilfe hat gegenüber einer früheren Auswertung von $70 \%$ auf $79 \%$ zugenommen. Gestiegen ist auch, von 25\% auf 30\%, der Anteil der hier festgestellten vorwerfbaren Behandlungsfehler. In der Mehrzahl waren es Vorwürfe zur Geburtsleitung, in $2 / 3$ der Fälle strittige vaginaloperative bzw. Schnittentbindungen. Zunehmend wird auch die präpartale Betreuung gerügt. Die seltene Komplikation der Schulterdystokie beinhaltet immerhin 18\% der Vorwürfe einer fehlerhaften Geburtsleitung; der Anteil der dabei als unzureichend betrachteten Maßnahmen sank von vormals 33 auf 20\%. Dem niedergelassenen geburtshilflich tätigen Frauenarzt galten jetzt $2 / 3$ statt früher $3 / 4$ der Vorwürfe zur Schwangerschaftsbetreuung, wobei im Vordergrund standen: Fehlbildungsdiagnostik, Wachstumsretardierung, Erkennen drohender Frühgeburtlichkeit bei Gestosesymptomatik, Plazentainsuffizienz sowie aufsteigender Genitalinfektionen. Die durchschnittliche Behandlungsfehlerquote der niedergelassenen Frauenärzte erhöhte sich im Berichtszeitraum auf $38 \%$.

Für die Qualitätssicherung lassen die Zahlen erkennen, dass in der Gynäkologie Fehler im Bereich der Onkologie, der operativen 
Diagnostik und Therapie und in der Geburtshilfe Fehler in der Geburtsleitung einschließlich der Behandlung der Schulterdystokie im Vordergrund stehen. Im niedergelassenen Bereich waren es Vorwürfe wegen fehlerhafter Schwangerschaftsbetreuung im Rahmen der pränatalen Diagnostik, Störungen der Kindsentwicklung und der sonographischen Missbildungsdiagnostik.

Fehler in der Eingriffsaufklärung wurden in 29\% der Fälle als haftungsbegründende Aufklärungsversäumnisse anerkannt, wobei häufig durch widersprechende Aussagen die Frage der Risikoaufklärung nicht abschließend beurteilt werden konnte. Ein lehrreicher Fall zur Risikoaufklärung:
Eine Frau wurde $1 / 2$ Stunde vor dem Eingriff nach Prä-Medikation mit Dormicum über eine Änderung und Erweiterung der geplanten Operation aufgeklärt. Die ursprünglich vaginal angesetzte Operation sollte nun abdominal erfolgen und eine Suspensionsplastik wegen Harninkontinenz durchgeführt werden. Bei großem Uterus myomatosus und Zeichen der Stressinkontinenz war die Indikation und Durchführung der so geänderten Operation richtig, die Operation war aber wegen unwirksamer Einwilligung rechtswidrig. Dies hat zur Folge, dass der behandelnde Arzt für alle im Zusammenhang mit dem Eingriff eingetretenen, d.h. auch für die nicht vermeidbaren gesundheitlichen Beeinträchtigungen haftungsrechtlich einstehen muss. 